Physical Sciences | Daniel Boyd

\section{An introduction to the information dimension}

The difficulty of defining the true nature of information has sparked a rich, seemingly unending variety of questions over the past centuries; from the nature of the human soul can gain consciousness. Now Daniel Boyd, an indesendent researcher in the Netherlands, believes that these problems could be solved if we view information as a substance residing in its own separate dimension, which exists in tandem with the physical world. The answers his understanding of our minds, as well as the capabilities of information technologies. profound implications have

\begin{abstract}
Ithough our consciousness is as
real to us as the physical objects
we interact with we interact with every day, its tenuous relationship with the physical world has kept philosophers engaged for centuries. According to Descartes, our minds are entirely separate from the physical world and yet linked to our bodies in some undefined way in since this is an entirely philosophical. concept that cannot be scientifically tested through experiment modern researchers find little satisfaction in Descartes' theory.

In the present day, the true nature of both information and consciousness continues to spark fierce, longstanding debates between physicists, neuroscientists and philosophers with an abundance of conflicting viewpoints and little progress towards a universal solution. Now, Daniel Boyd believes that this deadlock could finally be overcome by considering information a substance existing in its or
\end{abstract}

\section{NFORMATION AS A}

NON-PHYSICAL SUBSTANCE

Information is often considered to be an 'abstract' property, less real than as the charge of a particle or the mass of an object. However, Boyd argues that this isn't the case. To explain 'emere 'in which novel properties arise when different parts interact with each other. To take a very simple example, the forward motion of a bike can only occur when the bike and rider interact: neither can reproduce the overall behaviour on its own. Similarly, complex information phenomena must be the emergent product of interaction between information entities with more primitive properties. In order to interact, these entities, while not physical, must be real and act according to their counterparts, Boyd proposes the term 'quirk' for the most funda succincty these information entities. measurable physical properties such own laws. In line with their physical

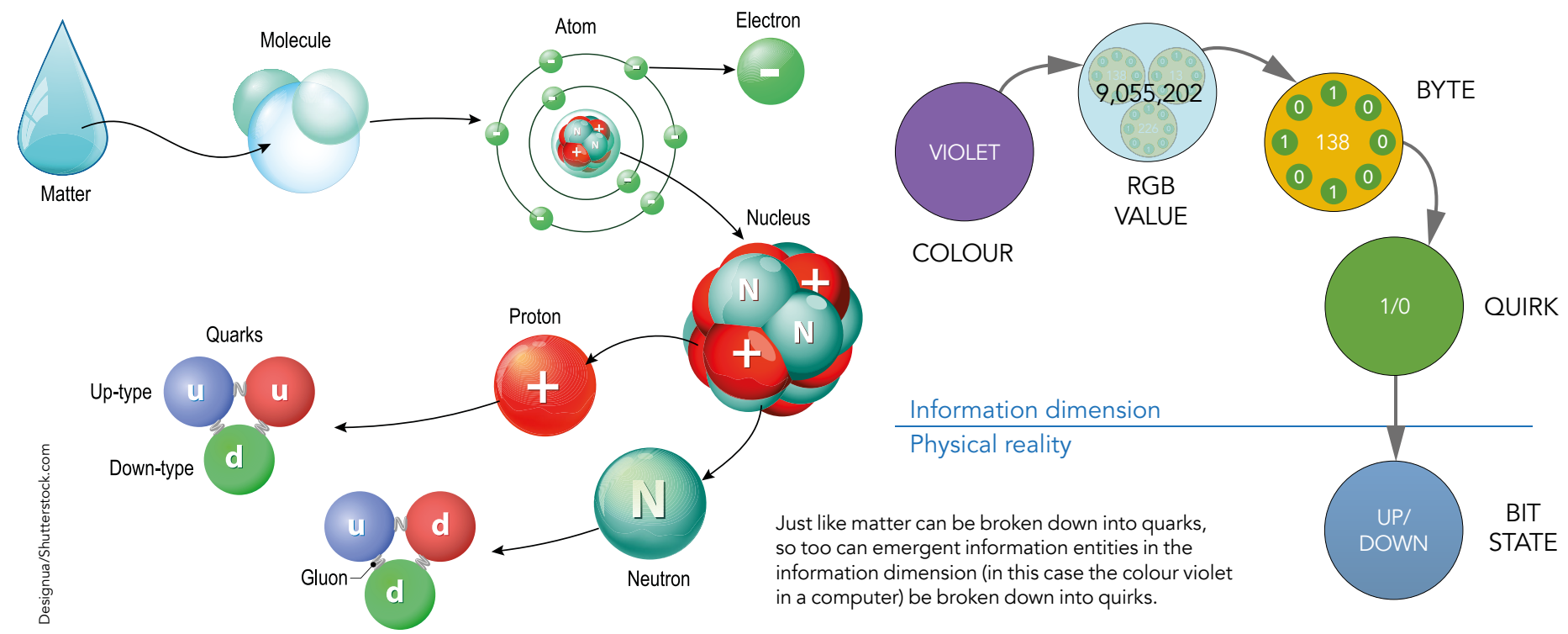

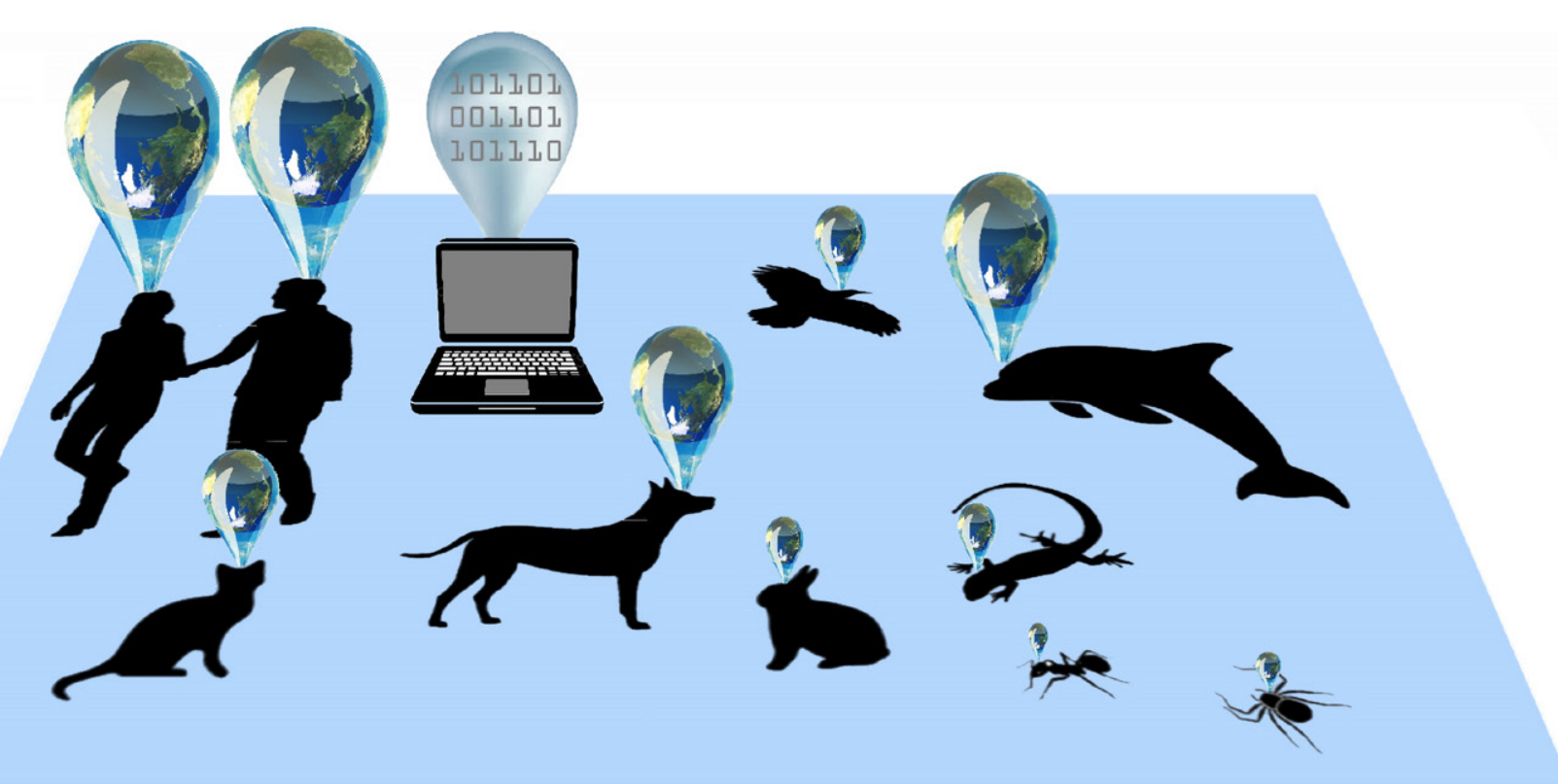

Boyd proposes a separate 'information dimension' that may be viewed as a 'bubble'. Inside this bubble, intormation processes play out and ultimately cause Proposing a new particle is rather Boyd suggests that a quirk is the

but extends outwards from our 3D reality, while remaining firmly attached simple fourth Euclidean dimension: n: the down to atoms, nuclei, nucleons, and finally, quarks. Each level has its own properties. Only at the top level does the entities interact. This changes the state of each participant in a way that is created by neural networks in particular property of solidity emerge.

related to the state of the other entity are highly complex.

Similarly, quirks in the information dimension can combine to form

an information is that it uses Boyd redefines the boundaries of reality sophisticated

to include an "information dimension". unique properties.

face value, but as a to include an "information dimension". As a simple example, representative of the a real, definable counterpart to the information about colour which is stored For instance, a physical dimensions. on a computer

computer reading a magnetic state binary value written into it.

\section{AN EXPANDED VIEW OF REALITY} Any real entity must exist somewhere, ities cannot exist in the physical world. To house them Boyd redefines the boundaries of reality to include an "information dimension" physical dimensions. Together, the two dimensions exhibit a 'substance dualism' which constitutes our reality.

Since our senses evolved to perceive the $3 \mathrm{D}$ world of matter and energy, it isn't easy for us to visualise such an additional dimension, but Boyd describesit as a bubble created by the brain to contan This bubble isn't a physical structure
Inside the information bubble, informational processes play out jus each other in the physical world. From humans to computers to ants, all systems whose characteristics are governed by information have such attached bubbles, and the information processes taking place within them intervene with their physical substrate to cause the behaviours we observe.

\section{HIERARCHICAL STRUCTURES} For this theory to work, Boyd argues tha the processes which occur within the information dimension must be as richly complex as those in the physical world notably, displaying clear hierarchical structures. A sold material, for example, maller builing blocks from mosively in the form of binany values which

combine to form bytes, RGB values, and finally, colour itself. In far more complex scenarios, billions of interacting quirks scales. Ultimately phenomena that are as different from elementary quirks as

CLEARING UP CONFUSION With so many other definitions and theories regarding information and consciousness already in existence, Boyd recognises the need to avoid confusion with other theories which dea with similar issues. Perhaps the most obvious risk arises from the apparent similarity between his substance dualism and Descartes mind-body theory, which gives the soul substance but falls to world in contact, Boyd shows how the 


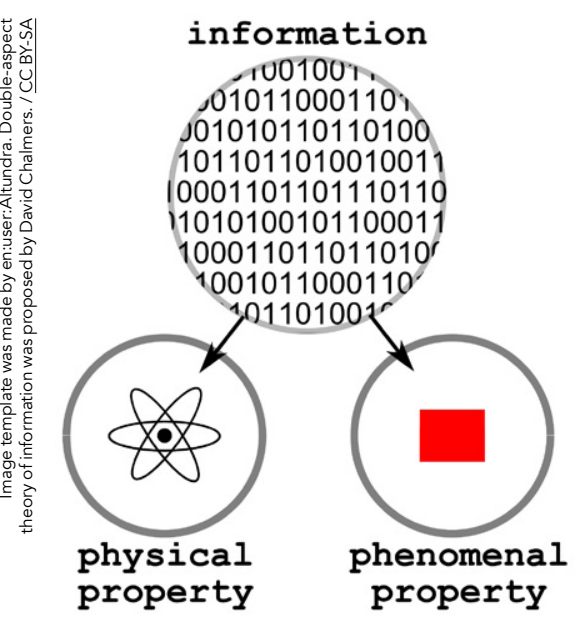

The 'double-aspect theory' defines
information as having both physical and information as having both physical and
conscius properties. This differs from the
theory Boyd put torward.

information dimension is firmly linked to the physical dimension through the physical substrate.

More recently, proponents of the 'double-aspect' theory of information have attempted to explain the influence of consciousness on the physical world by defining information as having both physical and conscious properties. This differs from Boyd's theory, which sees information as a separate substance, and consciousness as just one of a wide range of emergent information phenomena.

Boyd has incorporated elements from many existing fields, including information theory This makes it diffic al for sciextis theory. This makes it difficult for scientists confirm his theories, and may lead some academics to believe that Boyd's ideas conflict with theirs. Boyd doesn't consider this to be the case: instead, he believes that his theories provide useful, previous unseen links between these fields.

\section{FREEING THE WILL FROM} PHYSICAL DETERMINISM One of the most profound aspects of his research is that Boyd's theory provides a clear answer to the ancient question of whether humans really have free will - meaning that the outcomes of our decisions are not determined by physical laws. To the frustration of many philosophers, this would not be physical dimension.

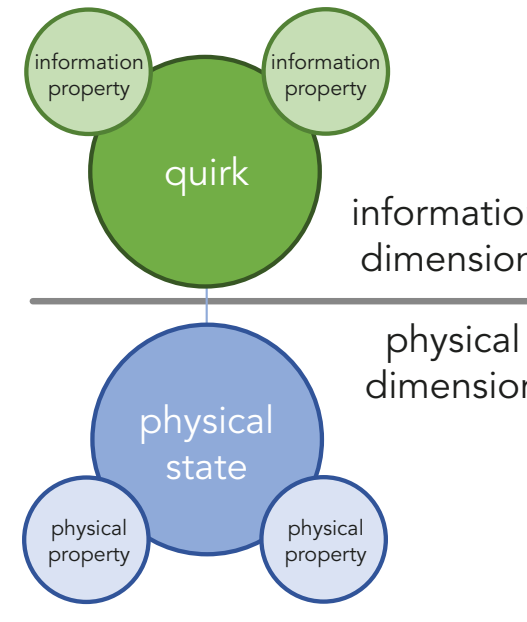

Rather than an abstract property of the physical world, boyd sees information as an
element of reality in its own right, existing in
its own dimension.

Boyd argues that if our conscious decision making takes place in the information dinension, it is indeed Furthermore, if our emergent minds top-down control over their component quirks, they can influence events in the physical world to which these quirks are connected. He suggests that brain evolved to allow for this influence, as it is necessary if they are to perform their someday be ethically obliged to trea these systems as sentient beings. Reassuringly, Boyd believes that his came about through our ownce Al design and not through evolution, it doesn't demonstrate the same organic self-organisation as our minds. The emergent phenomena operating within their information dimensions are therefore fundamentally different from those created by our brains. Even if Al produces identical output as our minds it is therefore mistaken to see this as evidence of consciousness. To do so is comparable to assuming that a tractor works using muscles, because we know pulling ploughs.

\section{THE WAY FORWARD}

By recognising the information ight rather than seeing infy in its ow merely as an abstract property of the physical world, Boyd believes that significant new avenues of research could be opened up. He acknowledges that in order to move beyond a purely theoretical framework, empirical evidence is needed. While what we

Even if Al produces identical output as our minds, it is mistaken to see this as evidence of consciousness.

and behaviours in our bodies on the basis of information processes. This frees but ironically, in doing so subiugates it to the equally deterministic laws operating in the information dimension.

\section{CONSEQUENCES FOR}

ARTIFICIAL INTELLIGENCE

Boyd's theories present a new angle

on one of the most pressing questions

facing artificial intelligence (A)): whether

these systems could one day becom

sophisticated enough to gain their

own consciousness. Using self-learning

algorithms and sensors to perceive the

surrounding environments, these systerns

are already capable of acting intelligen

in ways that sometimes mimic our own

improves, concerns grow that we may know about the workings of digital brains. As non-physical phenomena quirks and the emergent systems built from them cannot be detected using physical instruments. New techniques are therefore required which could one day provide researchers with rigorous, going on in the information dimension, consciousness. For now, he continues to develop his ideas through experiments involving cellular automata, which can be used as experimental models for neural networks. If his theories become more widely researched, they could break new ground in scientic and philosophical debas whic computers supports the theory, such testable explanations for everything including the specific properties of

\section{Behind the Research}

Daniel Boyd

E: daniel.boyd@live.n! T: +31 650431108

\section{Research Objectives}

In between the realms of information technology and neuroscience, Daniel Boyd is putting forward a theory of emergent information to explain both human mental capabilities and artificial intelligence.

\section{Detail}

Daniel Boyd
Brik 117

1625 GN Hoor

Netherlands

Bio

Daniel Boyd studied biology in the early 1980 s and for the existence and function of cons avilable biological systems. Since then he has been following and independently researching these topics, most recently with a focus on the emergent information in the brain.

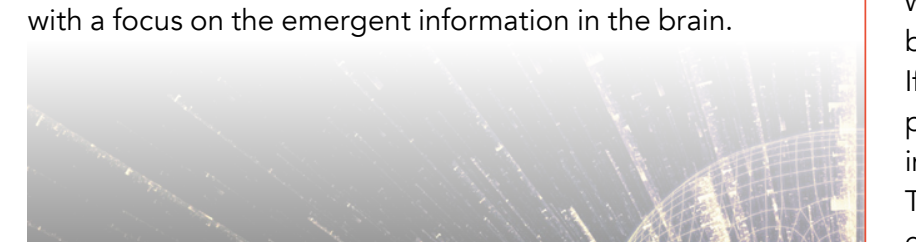

References

Boyd, D. (2020). Design and self-assembly of information Available at: https://doi.org/10.1080/03080188.2020.1712816

Chalmers, D. (1995). Facing up to the problem of consciousness. Journal of Consciousness Studies, $2(3)$, 200-219.

\section{Personal Response}

What are cellular automata, and how will experiments involving them help us to learn more about the information dimension?

II A cellular automaton is a grid of cells, each of which can mber of states which is determined through a set of rules relating to the states of its neighbours. This can be configured as a simplified simulation of a neural network, in which the states of individual neurons are also determined by the states of neighbours to which they are connected. designed to simulate self-organising information processes, it should be possible to demonstrate This wo emergence in biological neural networks.

II

